\title{
Distributed Opportunistic Scheduling For Ad-Hoc Communications Under Noisy Channel Estimation
}

\author{
Dong Zheng, Man-On Pun, Weiyan Ge, Junshan Zhang and H. Vincent Poor
}

\begin{abstract}
Distributed opportunistic scheduling is studied for wireless ad-hoc networks, where many links contend for one channel using random access. In such networks, distributed opportunistic scheduling (DOS) involves a process of joint channel probing and distributed scheduling. It has been shown that under perfect channel estimation, the optimal DOS for maximizing the network throughput is a pure threshold policy. In this paper, this formalism is generalized to explore DOS under noisy channel estimation, where the transmission rate needs to be backed off from the estimated rate to reduce the outage. It is shown that the optimal scheduling policy remains to be threshold-based, and that the rate threshold turns out to be a function of the variance of the estimation error and be a functional of the backoff rate function. Since the optimal backoff rate is intractable, a suboptimal linear backoff scheme that backs off the estimated signal-to-noise ratio (SNR) and hence the rate is proposed. The corresponding optimal backoff ratio and rate threshold can be obtained via an iterative algorithm. Finally, simulation results are provided to illustrate the tradeoff caused by increasing training time to improve channel estimation at the cost of probing efficiency.
\end{abstract}

\section{INTRODUCTION}

Channel-aware scheduling for achieving rich diversities inherent in wireless communications has recently emerged as a promising technique for improving spectral efficiency in wireless networks. Most existing studies along this line require centralized scheduling (see [5] and the references therein), and little work has been done on developing distributed algorithms to harvest diversity gains for ad hoc communications. Unlike centralized cases, in ad hoc communication networks, each link has no knowledge of other links' channel conditions, making it very challenging to carry out channel-aware distributed scheduling.

Some initial steps have been taken by several of the authors to develop distributed opportunistic scheduling (DOS) to reap multiuser diversity and time diversity in wireless ad hoc networks [7]. In DOS, a successful link proceeds to data transmission only if the observed channel condition is "good"; otherwise, it may skip the transmission, and let all the links recontend for the channel. Intuitively speaking, because different

Dong Zheng is with NextWave Wireless Inc., San Diego, CA 92130 (email: dzheng@nextwave.com).

Man-On Pun and H. Vincent Poor are with the Department of Electrical Engineering, Princeton University, Princeton, NJ 08544 (e-mail: mopun@princeton.edu; poor@princeton.edu).

Weiyan Ge and Junshan Zhang are with the Department of Electrical Engineering, Arizona State University, Tempe, AZ 85287 (e-mail: Weiyan.Ge@asu.edu; Junshan.Zhang@asu.edu).

This research was supported in part by the Croucher Foundation under a post-doctoral fellowship, in part by the U. S. National Science Foundation under Grants ANI-02-38550, ANI-03-38807, CNS-06-25637, and CNS-0721820 and in part by Office of Naval Research through Grant N00014-05-10636. links in different time slots experience different channel conditions, it is likely that after further probing, the channel can be taken by a link with a better channel condition, resulting in possible higher throughput. In this way, the multiuser diversity across links and the time diversity across slots can be exploited in a joint manner.

Despite the insightful analytical results reported in [7], its key assumption is that perfect channel state information (CSI) is known at the receiver/transmitter. However, in practical scenarios, channel conditions are often estimated using noisy observations. Therefore, it is of great interest to study DOS under noisy channel estimation. In centralized scheduling schemes, it has been shown that the estimated signal-to-noise ratio (SNR) is always larger than the "actual SNR" [6]. Thus, if the data were transmitted using the estimated rate, there would always be an outage. To reduce the outage probability, the transmission rate has to back off from the estimated rate. Therefore, unlike the perfect estimation case, the optimal scheduling policy hinges on the backoff rate.

In this work, we generalize [7] to the scenario with imperfect channel estimation and show that the optimal scheduling policy for the noisy channel estimation case remains a threshold structure. However, the threshold turns out to be a function of the variance of the channel estimation error, and furthermore, it is a functional of the backoff rate function. Since the optimal backoff rate function is difficult to obtain, we propose a suboptimal linear backoff scheme. We show that the corresponding optimal backoff ratio and rate threshold can be obtained via an iterative numerical algorithm. Simulation results are provided to show that DOS achieves significant throughput gain in the presence of noisy channel estimation, especially in the low SNR region.

\section{SyStem MODEL AND BACKGROUND}

We consider a single-hop ad hoc network with $M$ links, where link $m$ contends for the channel with probability $p_{m}$, $m=1, \ldots, M$. A collision model is assumed for random access, where channel contention of a link is said to be successful if no other links transmit at the same time. Accordingly, the overall successful contention probability, $p_{s}$, is then given by $\sum_{m=1}^{M}\left(p_{m} \prod_{i \neq m}\left(1-p_{i}\right)\right)$. It is clear that the number of slots (denoted as $K$ ) for a successful channel contention is a Geometric random variable (r.v.), i.e., $K \sim \operatorname{Geometric}\left(p_{s}\right)$. Let $\tau$ denote the duration of mini-slot for channel contention, and $T$ the data transmission time. It follows that the random duration corresponding to one round of successful channel contention is $K \tau$, with expectation $\tau / p_{s}$. 
Let $s(n)$ denote the successful link at the $n$-th successful channel contention. The corresponding received signal is given by:

$$
Y_{s(n)}(n)=\sqrt{\rho} h_{s(n)}(n) X_{s(n)}(n)+\mu_{s(n)}(n),
$$

where $\rho$ is the normalized receiver $\operatorname{SNR}, h_{s(n)}(n)$ is the channel coefficient for link $s(n), X_{s(n)}(n)$ is the transmitted signal with $E\left\{\left\|X_{s(n)}(n)\right\|^{2}\right\}=1$ and $\mu_{s(n)}(n)$ is additive white noise with i.i.d. $\mathcal{C N}(0,1)$.

To simplify the exposition, we consider a homogeneous network in which all links have the same channel statistics, and are subject to Rayleigh fading, i.e., $h_{s(n)}(n)$ follows a complex Gaussian distribution $\mathcal{C N}(0,1)$. In what follows, we drop the subscripts to simplify the notation and use $h_{n}$ to stand for $h_{s(n)}(n)$ where it is clear from the context. Similarly, we use $Y_{n}, X_{n}$ and $\mu_{n}$ to denote $Y_{s(n)}(n), X_{s(n)}(n)$ and $\mu_{s(n)}(n)$.

We consider the continuous rate case, assuming that the instantaneous rate is given by the Shannon channel capacity, i.e.,

$$
R_{n}=\log \left(1+\rho\left|h_{n}\right|^{2}\right) \text { nats } / \mathrm{s} / \mathrm{Hz},
$$

provided that the channel can be perfectly estimated.

In [7], we have studied DOS with perfect CSI. Specifically, we have shown that the problem can be cast as a maximal rate of return problem in optimal stopping theory [3], where the rate of return is the average network throughput, $x$, and is determined by the stopping time $N$ :

$$
x=\frac{E\left[R_{N} T\right]}{E\left[T_{N}\right]},
$$

where $T_{n} \triangleq \sum_{j=1}^{n} K_{j} \tau+T$ is the total system time including the contention time and the data transmission time. Note that $N$ is a stopping time if $\{N=n\}$ is $\mathcal{F}_{n}$-measurable, where $\mathcal{F}_{n}$ is the $\sigma$-field generated by $\left\{\left(\rho\left|h_{j}\right|^{2}, K_{j}\right), j=1,2, \ldots, n\right\}$.

We show that the optimal DOS maximizing the throughput is given by the optimal stopping rule, $N^{*}$, that solves the maximal rate of return problem in (2), i.e.,

$$
N^{*} \triangleq \underset{N \in Q}{\arg \max } \frac{E\left[R_{N} T\right]}{E\left[T_{N}\right]}, \quad x^{*} \triangleq \sup _{N \in Q} \frac{E\left[R_{N} T\right]}{E\left[T_{N}\right]},
$$

where

$$
Q \triangleq\left\{N: N \geq 1, E\left[T_{N}\right]<\infty\right\} .
$$

It has been shown in [7] that the optimal stopping rule $N^{*}$ for DOS exists, and is given by

$$
N^{*}=\min \left\{n \geq 1: R_{n} \geq x^{*}\right\} .
$$

Furthermore, the maximal throughput $x^{*}$ is an optimal threshold, and is the unique solution to

$$
E(R-x)^{+}=\frac{x \tau}{p_{s} T}
$$

where $R$ is a r.v. having the same distribution as $R_{n}$.

For example, if $R_{n}=\log \left(1+\rho\left|h_{n}\right|^{2}\right)$ and $h_{n}$ has a complex Gaussian distribution $\mathcal{C N}(0,1)$, it can be shown that

$$
x^{*}=\exp \left(\frac{1}{\rho}\right) E_{1}\left(\frac{\exp \left(x^{*}\right)}{\rho}\right) \frac{p_{s}}{\delta},
$$

where $\delta=\tau / T$, and $E_{1}(x)$ is the exponential integral function defined as $E_{1}(x) \triangleq \int_{x}^{\infty} \frac{\exp (-t)}{t} d t$.

\section{DOS Under NoISY ChanNEL Estimation}

Needless to say, in practical systems, $h_{n}$ has to be estimated using training signals (e.g. embedded in the RTS packets). Let $\hat{h}_{n}$ denote the estimation of the channel coefficient, and $\tilde{h}_{n}$ the estimation error. It follows that

$$
h_{n}=\hat{h}_{n}+\tilde{h}_{n},
$$

where $\hat{h}_{n}$ and $\tilde{h}_{n}$ are zero-mean complex Gaussian random variables. Suppose that the channel is estimated using a minimum mean square error (MMSE)-based estimator. It follows, by the orthogonality principle, that

$$
E\left[\left|h_{n}\right|^{2}\right]=E\left[\left|\hat{h}_{n}\right|^{2}\right]+E\left[\left|\tilde{h}_{n}\right|^{2}\right] .
$$

Let $\beta$ denote the variance of the estimation error. From (9), we have that

$$
E\left[\left|\tilde{h}_{n}\right|^{2}\right]=\beta \quad \text { and } \quad E\left[\left|\hat{h}_{n}\right|^{2}\right]=1-\beta .
$$

Treating the estimation error as noise, the actual SNR at the receiver can be computed by [6]

$$
\lambda_{n}=\frac{\rho\left|\hat{h}_{n}\right|^{2}}{1+\rho\left|\tilde{h}_{n}\right|^{2}} .
$$

We note that the numerator of $\left[11, \rho\left|\hat{h}_{n}\right|^{2}\right.$, is the estimated SNR. Therefore, in contrast to the perfect CSI case where the sequence $\left\{\rho\left|h_{n}\right|^{2}, n=1,2, \ldots\right\}$ is used for distributed scheduling, in the noisy estimation case, $\left\{\rho\left|\hat{h}_{n}\right|^{2}, n=\right.$ $1,2, \ldots\}$ serves as the basis for distributed scheduling.

Following [6], $\left|\hat{h}_{n}\right|^{2}$ and $\left|\tilde{h}_{n}\right|^{2}$ can be normalized as

$$
\hat{\lambda}_{n}=\frac{\left|\hat{h}_{n}\right|^{2}}{1-\beta} \quad \text { and } \quad z_{n}=\frac{\left|\tilde{h}_{n}\right|^{2}}{\beta} .
$$

Note that both $\hat{\lambda}_{n}$ and $z_{n}$ have the exponential distribution with unit variance. Furthermore, $\lambda_{n}$ in (11) can be rewritten as

$$
\lambda_{n}=\frac{\rho_{e f f} \hat{\lambda}_{n}}{1+\alpha \rho_{e f f} z_{n}},
$$

where $\rho_{\text {eff }} \triangleq(1-\beta) \rho$ and $\alpha \triangleq \frac{\beta}{1-\beta}$ denote the "effective channel SNR" and "normalized error variance", respectively. It can be shown the distribution of $\lambda_{n}$ given $\hat{\lambda}_{n}$ is given by [6]

$f\left(\lambda_{n} \mid \hat{\lambda}_{n}\right)=\frac{\hat{\lambda}_{n}}{\alpha \lambda_{n}^{2}} \exp \left\{-\frac{1}{\alpha}\left(\frac{\hat{\lambda}_{n}}{\lambda_{n}}-\frac{1}{\rho_{e f f}}\right)\right\} \mathbf{I}\left(\frac{\hat{\lambda}_{n}}{\lambda_{n}}-\frac{1}{\rho_{e f f}}\right)$,

where $\mathbf{I}(\cdot)$ is the indicator function.

\section{A. Optimal Stopping Rule under Noisy Channel Estimation}

It is clear that the actual SNR $\lambda_{n}$ is no greater than the estimated SNR $\rho_{e f f} \hat{\lambda}_{n}$. As a result, if the packet is transmitted at the estimated rate $\log \left(1+\rho_{e f f} \hat{\lambda}_{n}\right)$, there would always be a channel outage. Therefore, the transmission rate has to back off from the estimate rate. Equivalently, we can back 
off the estimated SNR $\rho_{\text {eff }} \hat{\lambda}$ to a "nominated" SNR $\lambda_{c}(\hat{\lambda})$. Accordingly, the instantaneous rate, $R_{n}$, is given by

$$
R_{n}=\log \left(1+\lambda_{c}\left(\hat{\lambda}_{n}\right)\right) \mathbf{I}\left(\lambda_{c}\left(\hat{\lambda}_{n}\right) \leq \lambda_{n}\right) .
$$

Along the same line as in the perfect CSI case, for each given back-off rate function $\lambda_{c}(\cdot)$, maximizing the average throughput reduces to solving the maximal rate of return problem in (2).

Observe that there are at least two major differences between the perfect estimation case and the noisy channel estimation case. First, the stopping rule $N$ is now defined over the $\sigma$-field $\mathcal{F}_{n}^{\prime}$ (instead of $\mathcal{F}_{n}$ ), generated by $\left\{\left(\rho\left|\hat{h}_{j}\right|^{2}, K_{j}\right), j=\right.$ $1,2, \ldots, n\}$. Second, the instantaneous rate, $R_{n}$, defined in (15), is now a random variable, and is not perfectly known at time $n$. However, it can be shown that the structure of the optimal scheduling strategy remains the same, except that the random "reward" $R_{n}$ is replaced with its conditional expectation, $\bar{R}_{n} \triangleq E\left[R_{n} \mid \mathcal{F}_{n}^{\prime}\right]$ [3, Page 1.3] [1]. More specifically, define

$$
Q^{\prime} \triangleq\left\{N \geq 1:\{N=n\} \in \mathcal{F}_{n}^{\prime}, E\left[T_{N}\right]<\infty\right\} .
$$

Analogously, define

$$
Q^{\prime \prime} \triangleq\left\{N \geq 1:\{N=n\} \in \mathcal{F}_{n}^{\prime \prime}, E\left[T_{N}\right]<\infty\right\},
$$

where $\mathcal{F}_{n}^{\prime \prime}$ is the $\sigma$-field generated by $\left\{\left(\bar{R}_{j}, K_{j}\right), j=\right.$ $1,2, \ldots, n\}$. We have the following proposition.

Proposition 3.1:

$$
\sup _{N \in Q^{\prime}} \frac{E\left[R_{N} T\right]}{E\left[T_{N}\right]}=\sup _{N \in Q^{\prime \prime}} \frac{E\left[\bar{R}_{N} T\right]}{E\left[T_{N}\right]} .
$$

The proof follows from Propositions 2.3, 2.4, 2.5 in [1] (with $X_{n}, Z_{n}, W_{n}$ in lieu of $R_{n}, \hat{\lambda}_{n}, \bar{R}_{n}$ ), and the proof in [7]. Due to space limitation, we omit the details here.

As a result, Proposition 3.1 indicates that the optimal scheduling can be based solely on $\bar{R}_{n}$, given by

$$
\begin{aligned}
\bar{R}_{n} & =E\left[R_{n} \mid \mathcal{F}_{n}^{\prime}\right] \\
& =\log \left(1+\lambda_{c}\left(\hat{\lambda}_{n}\right)\right)\left[1-\exp \left\{-\frac{1}{\alpha}\left(\frac{\hat{\lambda_{n}}}{\lambda_{c}\left(\hat{\lambda}_{n}\right)}-\frac{1}{\rho_{e f f}}\right)\right\}\right],
\end{aligned}
$$

where we have used the fact that $P\left(\lambda_{c}\left(\hat{\lambda}_{n}\right) \leq \lambda_{n} \mid \mathcal{F}_{n}^{\prime}\right)=$ $P\left(\lambda_{c}\left(\hat{\lambda}_{n}\right) \leq \lambda_{n} \mid \hat{\lambda}_{n}\right)$ due to the independence of channel estimations.

Based on the above discussion, we conclude that the optimal scheduling policy under noisy channel estimation is a pure threshold policy:

$$
N^{*}=\min \left\{n \geq 1: \bar{R}_{n} \geq x^{*}\right\},
$$

where the optimal threshold $x^{*}$ can be computed from (6), and hence, it is the unique solution to the following fixed point equation:

$$
x=\Phi\left(x, \lambda_{c}\right)
$$

where

$\Phi\left(x, \lambda_{c}\right) \triangleq \frac{\int_{\hat{\lambda}^{\prime}}^{\infty} e^{-\hat{\lambda}} \log \left(1+\lambda_{c}\right)\left[1-\exp \left\{-\frac{1}{\alpha}\left(\frac{\hat{\lambda}}{\lambda_{c}}-\frac{1}{\rho_{e f f}}\right)\right\}\right] d \hat{\lambda}}{\frac{\delta}{p_{s}}+e^{-\hat{\lambda}^{\prime}}}$ and $\hat{\lambda}^{\prime}$ can be obtained from

$$
\log \left(1+\lambda_{c}\left(\hat{\lambda}^{\prime}\right)\right)\left[1-\exp \left\{-\frac{1}{\alpha}\left(\frac{\hat{\lambda}^{\prime}}{\lambda_{c}\left(\hat{\lambda}^{\prime}\right)}-\frac{1}{\rho_{\text {eff }}}\right)\right\}\right]=x .
$$

\section{B. Optimal Backoff Rate Function}

It is clear from (20) that for a given backoff rate function $\lambda_{c}(\cdot)$, there is a corresponding optimal throughput $x^{*}$. Therefore, $x^{*}$ is a functional of $\lambda_{c}(\cdot)$, denoted as $x^{*}\left(\lambda_{c}\right)$. We are interested in finding the function $\lambda_{c}^{*}(\cdot)$ that maximizes $x^{*}\left(\lambda_{c}\right)$, i.e.,

$$
\lambda_{c}^{*}=\underset{\lambda_{c} \in A}{\arg \max } x^{*}\left(\lambda_{c}\right),
$$

where $A$ is the set of the admissible functions (for example, $A$ can be $\left.\left\{\lambda_{c}(\hat{\lambda}): \lambda_{c}(\hat{\lambda}) \geq 0, \forall \hat{\lambda} \geq 0\right\}\right)$.

Based on the theory of calculus of variations [4], problem (23) is a variational problem, and the functions $\lambda_{c}^{*}(\cdot)$ are called extremals. However, unlike the canonical calculus of variations problems, in this problem, the functional $x^{*}$ is not explicitly defined on $\lambda_{c}$. Instead, they are connected through a fixed point equation. Furthermore, the integral range in (21) is not fixed, but is a function of $\lambda_{c}$ (cf. (22)). As a result, it is intractable to characterize $\lambda_{c}^{*}$.

\section{A Suboptimal Backoff Rate Function}

In what follows, we propose a suboptimal backoff rate function, which backs off the estimated SNR by a multiplicative ratio $\sigma$, i.e., we set

$$
\lambda_{c}(\hat{\lambda})=\sigma \rho_{e f f} \hat{\lambda}
$$

and $0 \leq \sigma \leq 1$.

It follows from (24), (21) and (22) that the optimal throughput $x^{*}$ is the solution to

$$
\begin{aligned}
x & =\Phi(x, \sigma) \\
& =\left[1-\exp \left\{-\frac{1}{\alpha \rho_{e f f}}\left(\frac{1}{\sigma}-1\right)\right\}\right] \\
& \times \frac{\log \left(1+\sigma \rho_{e f f} \hat{\lambda}^{\prime}\right) e^{-\hat{\lambda}^{\prime}}+\exp \left(\frac{1}{\sigma \rho_{e f f}}\right) E_{1}\left(\hat{\lambda}^{\prime}+\frac{1}{\sigma \rho_{e f f}}\right)}{\frac{\delta}{p_{s}}+e^{-\hat{\lambda}^{\prime}}}
\end{aligned}
$$

where

$$
\hat{\lambda}^{\prime}=\frac{\exp \left(\frac{x}{1-\exp \left\{-\frac{1}{\alpha \rho_{\text {eff }}}\left(\frac{1}{\sigma}-1\right)\right\}}\right)-1}{\sigma \rho_{\text {eff }}} .
$$

It is not difficult to show that $x^{*}$ is a continuous and differentiable function of $\sigma$, and hence, there exists an optimal backoff ratio $\sigma^{*}$ such that

$$
\sigma^{*}=\underset{\sigma}{\arg \max } x^{*}(\sigma) .
$$

It can also be shown that $\sigma^{*}$ cannot be 0 or 1 (since the corresponding throughput is zero). Therefore, the optimal ratio $\sigma^{*}$ must satisfy the first order condition $\frac{d x^{*}\left(\sigma^{*}\right)}{d \sigma}=0$. 


\section{An Iterative Algorithm for Computing $\sigma^{*}$ and $x^{*}\left(\sigma^{*}\right)$}

Due to the complicated structure of the fixed point equation (25), it is not feasible to characterize $\sigma^{*}$ using the first order condition. In what follows, we devise an iterative algorithm instead using fractional optimization techniques [2].

Specifically, we define the following functions:

$U(\sigma, x) \triangleq\left[1-\exp \left\{-\frac{1}{\alpha \rho_{e f f}}\left(\frac{1}{\sigma}-1\right)\right\}\right] \times$

$\left\{\log \left(1+\sigma \rho_{e f f} \hat{\lambda}^{\prime}\right) \exp \left(-\hat{\lambda}^{\prime}\right)+\exp \left(\frac{1}{\sigma \rho_{\text {eff }}}\right) E_{1}\left(\hat{\lambda}^{\prime}+\frac{1}{\sigma \rho_{\text {eff }}}\right)\right\}$,

and $V(\sigma, x) \triangleq \frac{\delta}{p_{s}}+\exp \left(-\hat{\lambda}^{\prime}\right)$, where $\hat{\lambda}^{\prime}$ is defined in (26).

The iterative algorithm is outlined in Algorithm 1 , and its convergence is established in [8].

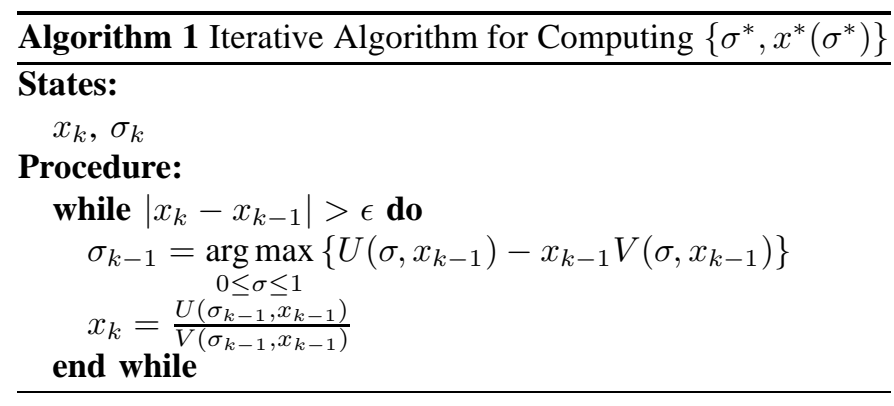

\section{Numerical Results}

In this section, we provide numerical examples to illustrate the above results. Unless otherwise specified, we assume that $\tau, T, p$, and $M$ are chosen such that $\delta=0.1$ and $p_{s}=$ $\exp (-1)$.

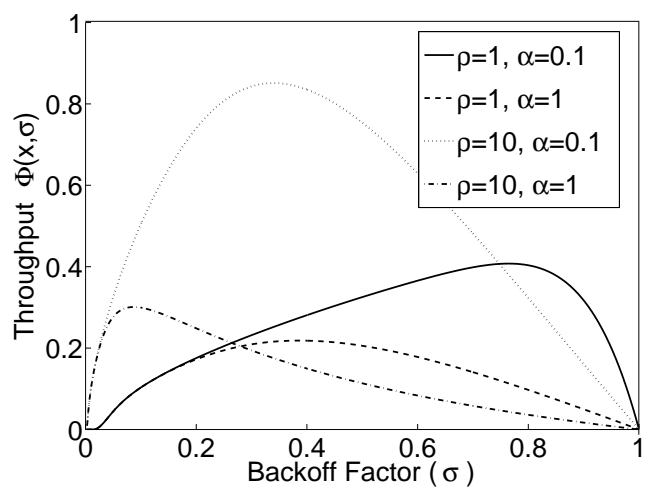

Fig. 1. $\Phi(\sigma)$ vs. $\sigma, x=0.1$.

Figure 1 depicts $\Phi(x, \sigma)$ as a function of the backoff ratio $\sigma$. It can be seen that the average throughput is zero at both $\sigma=0$ and $\sigma=1$, and is maximized somewhere in between.

Figure 2 depicts $\Phi\left(x, \sigma^{*}\right)$ as a function of $x$. Note that the optimal throughput $x^{*}$ is the solution to the fixed point equation $x=\Phi\left(x, \sigma^{*}\right)$. It can be observed that $x^{*}$ is an increasing function of $\rho$ for a given $\alpha$, and is a decreasing function of $\alpha$ for a fixed $\rho$. It can also be seen that the

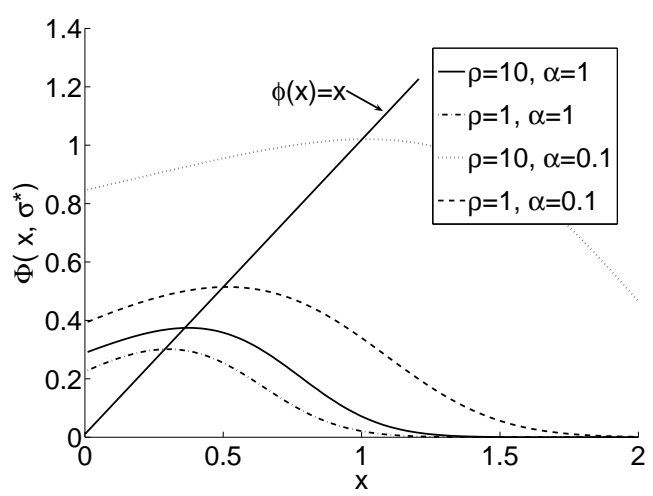

Fig. 2. $\Phi\left(x, \sigma^{*}\right)$ vs. $x$

estimation accuracy plays an important role in the throughput performance: when $\alpha$ decreases from 1 to 0.1 , the performance improves over $150 \%$ for $\rho=10$.

In Table I, we examine the convergence of the iterative algorithm I with $\alpha=1$. As expected, $x(n)$ approaches to $x^{*}$ usually within a few iterations.

TABLE I

CONVERGENCE BEHAVIOR OF THE ITERATIVE ALGORITHM, $\alpha=1$.

\begin{tabular}{|c|c|c|c|c|c|c|}
\hline$\rho$ & $x_{0}$ & $x_{1}$ & $x_{2}$ & $x_{3}$ & $x^{*}$ & $\sigma^{*}$ \\
\hline 0.5 & 0.5 & 0.177 & 0.246 & 0.254 & 0.254 & 0.407 \\
\hline 1 & 0.5 & 0.254 & 0.299 & 0.301 & 0.301 & 0.285 \\
\hline 2 & 0.5 & 0.306 & 0.335 & 0.336 & 0.336 & 0.182 \\
\hline 5 & 0.5 & 0.344 & 0.363 & 0.364 & 0.364 & 0.090 \\
\hline 10 & 0.5 & 0.358 & 0.374 & 0.374 & 0.374 & 0.049 \\
\hline
\end{tabular}

Table I compares the convergence behavior of the iterative algorithm with different error variance $\alpha$ and $\rho=1$. When the error variance is large, the iterative algorithm needs more iterations to converge. Moreover, the backoff ratio $\sigma$ would decrease as $\alpha$ increases. This can be further observed in Fig. 3 It indicates that when the estimation error is large, the transmitter would back off more to avoid channel outage.

TABLE II

CONVERGENCE BEHAVIOR OF THE ITERATIVE ALGORITHM, $\rho=1$.

\begin{tabular}{|c|c|c|c|c|c|c|c|c|}
\hline$\alpha$ & $x_{0}$ & $x_{1}$ & $x_{2}$ & $x_{3}$ & $x_{4}$ & $x_{5}$ & $x^{*}$ & $\sigma^{*}$ \\
\hline 0 & 0.5 & 0.604 & 0.610 & & & & 0.610 & 1.00 \\
\hline 0.1 & 0.5 & 0.514 & 0.514 & & & & 0.514 & 0.753 \\
\hline 1 & 0.5 & 0.254 & 0.299 & 0.301 & & & 0.301 & 0.285 \\
\hline 2 & 0.5 & 0.109 & 0.201 & 0.217 & 0.218 & & 0.218 & 0.155 \\
\hline 5 & 0.5 & 0.004 & 0.091 & 0.120 & 0.122 & 0.123 & 0.123 & 0.054 \\
\hline
\end{tabular}

Table IIII illustrates the throughput gain, $g=\frac{x^{*}-x^{L}}{x^{L}}$, as a function of $\rho$, where $x^{L}=\Phi\left(0, \sigma^{*}\right)$ is the average throughput obtained by the schemes without using optimal scheduling. It can be seen that the throughput gain is more significant in the low SNR region, and is a decreasing function of $\rho$.

In Table IV] we illustrate the throughput gain as a function of $\alpha$. Note that $\alpha=1 /(1-\beta)-1$ is an increasing function of $\beta$. 


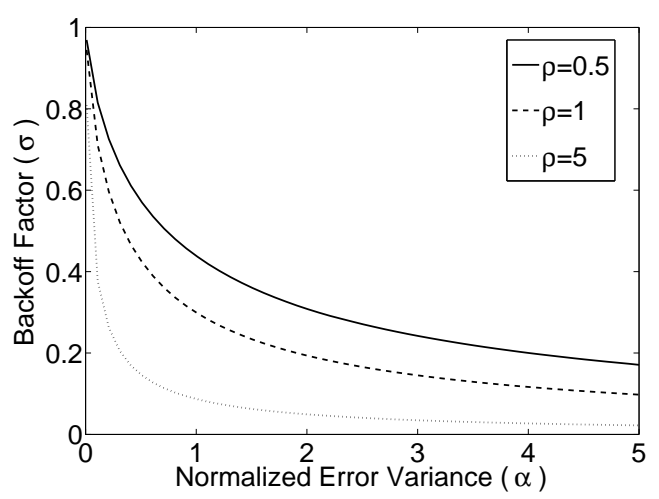

Fig. 3. Backoff factor $\sigma$ as a function of normalized error variance $\alpha$

TABLE III

THROUGHPUT GAIN OF DOS, $\alpha=1$.

\begin{tabular}{|c|c|c|c|c|c|c|}
\hline$\rho$ & 0.5 & 1 & 2 & 5 & 10 & 100 \\
\hline$x^{*}$ & 0.254 & 0.301 & 0.336 & 0.364 & 0.374 & 0.385 \\
\hline$x^{L}$ & 0.185 & 0.224 & 0.254 & 0.278 & 0.288 & 0.298 \\
\hline$g(\rho)$ & $37.3 \%$ & $34.3 \%$ & $32.3 \%$ & $30.9 \%$ & $29.8 \%$ & $29.2 \%$ \\
\hline
\end{tabular}

As expected, when the normalized noise variance $\alpha$ increases, the optimal throughput $x^{*}$ decreases, as well as the $x^{L}$. However, it is interesting to observe that the throughput gain increases instead. The rationale behind is that the performance of the schemes that do not use optimal scheduling "suffers" more than that of the distributed opportunistic scheduling in the presence of noisy channel estimation.

TABLE IV

THROUGHPUT GAIN OF DOS, $\rho=0.5$.

\begin{tabular}{|c|c|c|c|c|c|c|}
\hline$\alpha$ & 0 & 0.01 & 0.1 & 1 & 2 & 5 \\
\hline$x^{*}$ & 0.384 & 0.378 & 0.352 & 0.254 & 0.197 & 0.118 \\
\hline$x^{L}$ & 0.284 & 0.279 & 0.259 & 0.186 & 0.143 & 0.085 \\
\hline$g(\alpha)$ & $35.2 \%$ & $35.5 \%$ & $35.9 \%$ & $36.6 \%$ & $37.8 \%$ & $38.8 \%$ \\
\hline
\end{tabular}

We also examine the performance of distributed opportunistic scheduling as a function of the training time $\tau$. According to linear estimation theory, it has been shown that the error variance $\beta$ and the training time $\tau$ have the following relationship [8]:

$$
\beta=\frac{1}{\rho \tau+1} .
$$

Using (28) in the simulation, we plot throughput performance of the distributed opportunistic scheduling as a function of the training time $\tau$ in Fig. 4. It is clear that there exists an optimal training time which balances the tradeoff between better estimation accuracy and loss of transmission time. It can also be observed that when the average $\operatorname{SNR} \rho$ increases, the optimal training time decreases.

\section{CONClusion}

In this paper, we have generalized the study in [7] to investigate distributed opportunistic scheduling under noisy

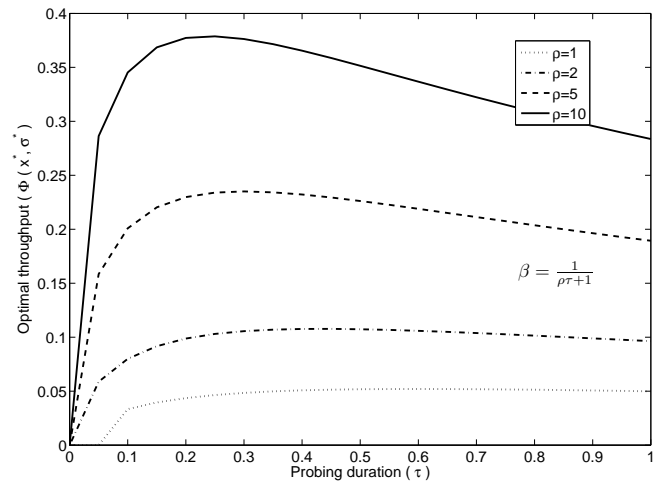

Fig. 4. Throughput vs. training time $\tau$

channel estimation. For such cases, we have proposed that the transmission rate backs off on the estimated rate so as to reduce the outage probability. We have showed that the optimal scheduling policy has a threshold structure, but the threshold turns out to be a function of the variance of the estimation error, and is a functional of the backoff rate function. Since the optimal backoff is analytically intractable, we have proposed a suboptimal linear scheme that backs off on the estimated SNR (and hence the rate). The corresponding optimal backoff ratios and rate thresholds can be obtained using an iterative algorithm based on fractional maximization. Simulation results indicate that DOS still achieves significant throughput gain in the presence of noisy channel estimation, especially in the low SNR region. In addition, we have observed that the performance loss of DOS due to the imperfect channel estimation is less than that of the schemes that do not use channel-aware scheduling, indicating that the devised DOS is more robust against noisy channel estimation.

\section{REFERENCES}

[1] D. Assal, L. Goldstein, and E. Samuel-Cahn, "A statistical version of prophet inequalities," Annals of Statistics, vol. 26, pp. 1190-1197, 1998.

[2] D. P. Bertsekas, Nonlinear Programming. Belmont, MA: Athena Scientific, 1995.

[3] T. Ferguson, Optimal Stopping and Applications. available at http://www.math.ucla.edu/ tom/Stopping/Contents.html, 2006.

[4] I. Gelfand and S. Fomin, Calculus of Variations. Englewood Cliffs, NJ: Prentice-Hall, 1963.

[5] X. Qin and R. Berry, "Exploiting multiuser diversity for medium access control in wireless networks," in Proc. IEEE INFOCOM'03, San Francisco, CA, Mar. 2003.

[6] A. Vakili, M. Sharif, and B. Hassibi, "The effect of channel estimation error on the throughput of broadcast channels," in Proc. IEEE Int'l Conf. Acoust. Speech Signal Process, Toulouse, France, May 2006.

[7] D. Zheng, W. Ge, and J. Zhang, "Distributed opportunistic scheduling for ad-hoc communications: An optimal stopping approach," in Proc. ACM Mobihoc 2007, Montreal, Canada, Sep. 2007.

[8] D. Zheng, M.-O. Pun, W. Ge, J. Zhang, and H. V. Poor, "Distributed opportunistic scheduling for ad hoc communications with imperfect channel information," Submitted to IEEE Transactions on Wireless Communications, 2007. 